\section{OP-37 UNPACKING 'EVIDENCE' IN EVIDENCE-BASED PUBLIC HEALTH POLICY: CAUTIONS AND CONSTRAINTS}

Adithya Pradyumna, Prasanna Saligram. SOCHARA - Society for Community Health Awareness Research and Action, Bangalore (Karnataka), India

\subsection{6/bmjgh-2016-EPHPabstracts.37}

Background Today, there is increasing emphasis on evidencebased public health policy, stepping away from a scenario where eminence and tokenistic appeasement play a major role in policymaking. In India since independence, there have been efforts towards evidence-informed policymaking through the Planning Commission as well as through health task forces at national and state levels. Evaluation of some public health programmes has also taken place both internally and externally. However, it would be important to closely and carefully examine how the use of evidence in policymaking plays out in the current institutional context.

Methods The policy triangle framework as proposed by Walt and Gilson ${ }^{1}$ was used to understand various aspects of the health policy experience from India. The framework puts policy content, process and context at the three corners of the triangle and policy actors in the centre. Social justice and public health ethics perspectives were employed to further unpack the various aspects of the policy triangle. The emerging issues from this qualitative analytical exercise were grouped together into overarching themes defining key cross-cutting challenges in use of evidence in policymaking processes in resource constrained settings.

Findings While use of evidence in policymaking processes can take place in a most desirable way when appropriate evidence is generated and when policymakers and institutions are primed for critiquing and adopting such evidence, our examination of prevailing research and policy institutions demonstrated that they are not in optimal position to fulfil such roles in the present context of power dynamics, disciplinary boundaries and inadequate capacity.

As for power dynamics, power plays out at different levels and between groups: between the decision maker and the people, between the researcher and the researched. In that context, along with the emphasis on evidence-based decisionmaking, it is important to reflect on who generates the evidence. More specifically, how sensitive is the researcher to power dynamics and to socio-political structures. The lived experience of persons from marginalised groups was seen to be often neglected. This relates to the question of 'what is meant by evidence?'. There was no clarity on the extent of ethical and social justice considerations made while choosing to use specific evidence and the resultant policy implications. In addition, some types of evidence were considered more 'powerful' within decision-making circles irrespective of whether the research study design was appropriate for answering the questions. For example, randomised controlled trials and economic evaluations had greater weightage over qualitative evidence and other social science methods. Instances of 'evidence gathering' commissioned by groups with vested interests abound including cherry picking of evidence to favour group's interests. Such practices were observed in case of public health interventions related to nutrition enhancement and control of infectious diseases.

As for disciplinary boundaries, public health - by nature - is an interdisciplinary field and interdepartmental sector. Population health would only improve by including health concerns as part of policies across sectors. However, related departments often hesitate to step into one another's 'territory'. Furthermore, individuals can't claim to have comprehensive knowledge in public health. Evidence is usually built upon a frame, which provides only part of the whole picture. Policies constructed through partial evidence base could lead to tokenistic policies, which either marginally improve the situation or in some cases may worsen it. They usually also do not address the status quo. The boundary issues may also have contributed to the present domination of the biomedical perspective in evidence generation, as biomedicine remains a major player in the sector.

As for inadequate capacity, the quality of evidence available is most often poor. Poor capacity for decision-making in the context of poor quality evidence emerged as a key concern. Any uncertainties and complexities may need to be dealt with caution. The capacity of translating the evidence into policy is also inadequate, for instance, interpreting the evidence with regard to the context, the ethical dimensions, and the resultant implications of the policy change. Finally, with ever-increasing information, the capacity to digest all relevant information and allow timely revision to policy is also a constraint.

Conclusions The call for evidence-based policy is a statement of intent and not necessarily a statement of action. There is need for reform within implementing institutions to be able to harness evidence appropriately based on technical capacity, ethics and integrity. Similarly, there is need for capacity building on the research front with regard to policy engagement, political analysis and ethical critique. There is need for a platform for the engagement of policymakers, scientists and health workers to deliberate on evidence. While healthcare companies have their lobby groups, countervailing power within academia and civil society need to be strengthened and/or alternative mechanisms for balanced dialogue need to emerge. The public health goal of improving health status and wellbeing can only be achieved through intersectoral coordination and action. Healthin-All policy is a technically feasible approach towards this. 
Systems approach would be useful towards understanding larger determinants of health services and health outcomes. Useful evidence can only be generated if programmes are well grounded and function over a period of several years. This, in turn, is possible if health policies are visions for the long term and programmes are not interrupted by changes in political regimes.

No competing interest.

\section{REFERENCE}

1 Walt G, Gilson L. Reforming the health sector in developing countries: the central role of policy analysis. Health Policy Plann 1994;9:353-70. 